\title{
Second Order Sufficient Optimality Conditions for Continuous-Time Programming Problems with Equality Constraints
}

Moisés Rodrigues Cirilo do Monte ${ }^{1}$

Instituto de Ciências Exatas e Naturais do Pontal - UFU, Ituiutaba - MG

Valeriano Antunes de Oliveira ${ }^{2}$

Instituto de Biociências, Letras e Ciências Exatas - UNESP, São José do Rio Preto - SP

\begin{abstract}
Second order sufficient optimality conditions are established for continuoustime optimization problems with equality constraints. The approach consists in obtaining second-order sufficient conditions for unconstrained problems first, transforming the equality constrained problem into an unconstrained one through penalization and then applying the first result.

keywords. Continuous-Time Programming Problems, Equality Constraints, Sufficient Conditions.
\end{abstract}

\section{Introduction}

We are concerned with the nonlinear continuous-time optimization problem with equality constraints in the form

$$
\begin{array}{ll}
\text { maximize } & P(z)=\int_{0}^{T} \phi(z(t), t) d t \\
\text { subject to } & h(z(t), t)=0 \text { a.e. } t \in[0, T], \\
& z \in L_{\infty}\left([0, T] ; \mathbb{R}^{n}\right),
\end{array}
$$

where $\phi: \mathbb{R}^{n} \times[0, T] \rightarrow \mathbb{R}$ and $h: \mathbb{R}^{n} \times[0, T] \rightarrow \mathbb{R}^{p}$. Here, $L_{\infty}\left([0, T] ; \mathbb{R}^{n}\right)$ denotes the Banach space of all Lebesgue-measurable essentially-bounded $n$-dimensional vector functions defined on the compact interval $[0, T] \subset \mathbb{R}$, with the norm $\|\cdot\|_{\infty}$ defined by

$$
\|z\|_{\infty}=\max _{1 \leq i \leq n} \operatorname{esssup}_{t \in[0, T]}\left|z_{i}(t)\right|
$$

All vectors are column vectors, unless transposed when they will be denoted by a prime, and all integrals are in the Lebesgue sense.

\footnotetext{
${ }^{1}$ moises.monte@ufu.br.

${ }^{2}$ valeriano.oliveira@unesp.br.
} 
Continuous-time problems arise often in the literature and were first proposed by Bellman [1] in his studies of some dynamic models of production and inventory called "bottleneck processes", which gave rise to continuous-time linear programming. Such problems contained only inequality constraints and can be written in the form

$$
\begin{array}{cl}
\text { maximize } & P(z)=\int_{0}^{T} \phi(z(t), t) d t \\
\text { subject to } & g(z(t), t) \geq 0, \text { a.e. } t \in[0, T], \\
& z \in L_{\infty}\left([0, T] ; \mathbb{R}^{n}\right),
\end{array}
$$

where $g: h: \mathbb{R}^{n} \times[0, T] \rightarrow \mathbb{R}^{m}$. For a summary of the results pertaining to necessary optimality conditions in continuous-time problems with inequality constraints and duality, with a fairly extensive list of relevant references, the reader is referred to $[3,7,8]$.

Several authors presented sufficient optimality conditions for certain classes of continuoustime problems with non-linear inequality constraints, for example, Rojas-Medar, Brandão and Silva [2], Oliveira and Rojas-Medar [6] using hypotheses of generalized convexity and Zalmai [9] which considered a problem with equality and inequality, but assuming hypotheses of pseudoconvexity on the problem.

The main motivation of this work lies in the fact that, in the formulation given in (1) with equality constraints, without hypotheses of convexity on the problem and the feasible solutions belonging to $L_{\infty}\left([0, T] ; \mathbb{R}^{n}\right)$, sufficient optimality conditions are not found in the literature. Besides, the problem (1) contains certain classes of constrained variational and optimal control problems and can be used to solve them both from the theoretical and computational point of view, serving as the basis for algorithms architecture.

The text is organized as follows. In Section 2, we give some preliminaries and the sufficient optimality conditions for the unconstrained problem. In Section 3, using previous results, the problem (1) is treated and we present an example. Final comments are given in Section 4.

\section{Unconstrained Problem}

Denote the feasible set of problem (1) by

$$
\Omega=\left\{z \in L_{\infty}\left([0, T] ; \mathbb{R}^{n}\right) \mid h(z(t), t)=0 \text { a.e. }[0, T]\right\}
$$

and for $z \in \Omega$, denote $\nabla h(z(t), t)=\left(\nabla h_{1}(z(t)), \ldots, \nabla h_{p}(z(t), t)\right)^{\prime}$ a.e. $t \in[0, T]$.

Definition 2.1. We say that $\bar{z} \in \Omega$ is a local optimal solution of problem (1) if there exists $\epsilon>0$ such that $P(\bar{z}) \geq P(z)$ for all $z \in \Omega$ satisfying $\|z-\bar{z}\|_{\infty}<\epsilon$.

Suppose the following assumptions valid:

(H1) $\phi(\cdot, t)$ is continuously differentiable throughout $[0, T], \phi(z, \cdot)$ and $\nabla \phi(z, \cdot)$ are Lebesgue measurable for each $z$, and there exists a number $K_{\phi}>0$ such that

$$
\|\nabla \phi(\bar{z}(t), t)\| \leq K_{\phi} \text { a.e. in }[0, T] .
$$


(H2) $h(\cdot, t)$ is continuously differentiable throughout $[0, T]$ and $h(z, \cdot)$ and $\nabla h(z, \cdot)$ are Lebesgue measurable for each $z$. There exists $K_{1}>0$ such that

$$
\|\nabla h(\bar{z}(t), t)\| \leq K_{1} \text { a.e. } t \in[0, T]
$$

Consider the unconstrained problem

$$
\begin{array}{ll}
\text { maximize } & P(z)=\int_{0}^{T} \phi(z(t), t) d t \\
\text { subject to } & z \in L_{\infty}\left([0, T] ; \mathbb{R}^{n}\right),
\end{array}
$$

and assume that (H1) holds. In the next proposition, we derive sufficient optimality conditions for the problem (2).

Proposition 2.1. If the assumptions

(a) $\nabla \phi(\bar{z}(t), t)=0$ a.e. $t \in[0, T]$, and

(b) $\int_{0}^{T} \gamma(t)^{\prime} \nabla^{2} \phi(\bar{z}(t), t) \gamma(t) d t<0 \forall \gamma \in L_{\infty}\left([0, T] ; \mathbb{R}^{n}\right) \backslash\{0\}$

hold, then $\bar{z}$ is a local optimal solution for the problem (2).

Proof. Suppose that $\bar{z}$ is not a local optimal solution for (2). Then there exists $\bar{\gamma} \in$ $L_{\infty}\left([0, T] ; \mathbb{R}^{n}\right) \backslash\{0\}$ and a number $\bar{\tau}>0$ such that

$$
P(\bar{z}+\tau \bar{\gamma})>P(\bar{z}), \tau \in(0, \bar{\tau})
$$

By the second order Taylor expansion in Banach Spaces (see [5]) we have that

$$
0<P(\bar{z}+\tau \bar{\gamma})-P(\bar{z})=\tau \delta P(\bar{z} ; \bar{\gamma})+\frac{1}{2} \tau^{2} \delta^{2} P(\bar{z} ;(\bar{\gamma}, \bar{\gamma}))+\epsilon\left(\tau^{2}\right)
$$

where $\epsilon\left(\tau^{2}\right) / \tau^{2} \rightarrow 0$ when $\tau \rightarrow 0$. Using the hypothesis $(a)$, it results that

$$
\delta P(\bar{z} ; \bar{\gamma})=\int_{0}^{T} \nabla \phi(\bar{z}(t), t)^{\prime} \bar{\gamma}(t) d t=0 .
$$

Therefore, (3) implies in

$$
0<\frac{1}{2} \tau^{2} \delta^{2} P(\bar{z} ;(\bar{\gamma}, \bar{\gamma}))+\epsilon\left(\tau^{2}\right)
$$

where $\epsilon\left(\tau^{2}\right) / \tau^{2} \rightarrow 0$ when $\tau \rightarrow 0$. Dividing both sides of (4) and taking limits as $\tau \rightarrow 0$, we obtain

$$
\delta^{2} P(\bar{z} ;(\bar{\gamma}, \bar{\gamma}))=\int_{0}^{T} \bar{\gamma}(t)^{\prime} \nabla^{2} \phi(\bar{z}(t), t) \bar{\gamma}(t) d t \geq 0
$$

contradicting $(b)$. 


\section{Problem with Equality Constraints}

Now, we will consider the problem (1). We assume throughout this section that (H1) and (H2) hold. The next result will be useful in the proof of sufficient optimality conditions for the problem (1).

Lemma 3.1. Let $R, S:[0, T] \rightarrow \mathbb{R}^{n \times n}$ be symmetric matrices with measurable entries. Suppose that

$$
\int_{0}^{T} \gamma(t)^{\prime} S(t) \gamma(t) d t=0, \quad \forall \gamma \in L_{\infty}\left([0, T] ; \mathbb{R}^{n}\right) \backslash\{0\} \Rightarrow \int_{0}^{T} \gamma(t)^{\prime} R(t) \gamma(t) d t<0,
$$

(B)

$$
\int_{0}^{T} \gamma(t)^{\prime} S(t) \gamma(t) d t \leq 0 \quad \forall \gamma \in L_{\infty}\left([0, T] ; \mathbb{R}^{n}\right) .
$$

Then there exists a scalar $\bar{\alpha}>0$ such that

$$
\int_{0}^{T} \gamma(t)^{\prime} R(t) \gamma(t) d t+\alpha \int_{0}^{T} \gamma(t)^{\prime} S(t) \gamma(t) d t<0
$$

for all $\gamma \in L_{\infty}\left([0, T] ; \mathbb{R}^{n}\right) \backslash\{0\}$ and $\alpha>\bar{\alpha}$.

Proof. By contradiction, assume that there exists a sequence $\left\{\gamma^{k}\right\}_{k \in \mathbb{N}} \subset L_{\infty}\left([0, T] ; \mathbb{R}^{n}\right) \backslash$ $\{0\},\left\|\gamma^{k}\right\|_{\infty}=1$ for all $k$, such that

$$
\int_{0}^{T} \gamma^{k}(t)^{\prime} R(t) \gamma^{k}(t) d t+k \int_{0}^{T} \gamma^{k}(t)^{\prime} S(t) \gamma^{k}(t) d t \geq 0 .
$$

Since the sequence $\left\{\gamma^{k}\right\}_{k \in \mathbb{N}}$ is bounded in $L_{\infty}\left([0, T] ; \mathbb{R}^{n}\right) \backslash\{0\}$, there exists a subsequence $\left\{\gamma^{k}\right\}_{k \in \mathbb{N}^{\prime}}, \mathbb{N}^{\prime} \subset \mathbb{N}$, converging weakly to a function $\bar{\gamma} \in L_{\infty}\left([0, T] ; \mathbb{R}^{n}\right) \backslash\{0\}$ (see [4]). Then, in (5) we have that

$$
\begin{aligned}
& \int_{0}^{T} \gamma^{k}(t)^{\prime} R(t) \gamma^{k}(t) d t \stackrel{k \rightarrow \infty}{\longrightarrow} \int_{0}^{T} \bar{\gamma}(t)^{\prime} R(t) \bar{\gamma}(t) d t, \\
& \int_{0}^{T} \gamma^{k}(t)^{\prime} S(t) \gamma^{k}(t) d t \stackrel{k \rightarrow \infty}{\longrightarrow} \int_{0}^{T} \bar{\gamma}(t)^{\prime} S(t) \bar{\gamma}(t) d t .
\end{aligned}
$$

From hypothesis $(B)$, we have that

$$
\int_{0}^{T} \bar{\gamma}(t)^{\prime} S(t) \bar{\gamma}(t) d t \leq 0
$$

and observing (5) we conclude that

$$
\int_{0}^{T} \bar{\gamma}(t)^{\prime} S(t) \bar{\gamma}(t) d t=0
$$


for otherwise the left-hand side in (5) would be $-\infty$. Therefore, from (5) we conclude that

$$
\int_{0}^{T} \bar{\gamma}(t)^{\prime} R(t) \bar{\gamma}(t) d t \geq 0
$$

contradicting hypothesis (A).

We are now in position to state and prove the main result of this section: the sufficient optimality conditions for problem (1).

Theorem 3.1. Let $\bar{z} \in \Omega$ and suppose that there exists $u^{*} \in L_{\infty}\left([0, T] ; \mathbb{R}^{p}\right)$ such that

(i) $\nabla \phi(\bar{z}(t), t)+\nabla h(\bar{z}(t), t)^{\prime} u^{*}(t)=0$ a.e. $t \in[0, T]$ and

(ii) $\int_{0}^{T} \gamma(t)^{\prime}\left\{\nabla^{2} L(\bar{z}(t), t)\right\} \gamma(t) d t<0$ for all $\gamma \in N \backslash\{0\}$, where

$$
L(z(t), t)=\phi(z(t), t)+\sum_{i=1}^{p} u_{i}^{*}(t) h_{i}(z(t), t) \quad \text { a.e. } t \in[0, T]
$$

and

$$
N=\left\{\gamma \in L_{\infty}\left([0, T] ; \mathbb{R}^{n}\right) \mid \nabla h(\bar{z}(t), t)^{\prime} \gamma(t)=0 \text { a.e. } t \in[0, T]\right\} .
$$

Proof. Consider the unconstrained auxiliary problem

$$
\begin{array}{ll}
\text { maximize } & \tilde{P}(z)=\int_{0}^{T} L_{\alpha}(z(t), t) d t \\
\text { subject to } & z \in L_{\infty}\left([0, T] ; \mathbb{R}^{n}\right),
\end{array}
$$

where $L_{\alpha}: \mathbb{R}^{n} \times[0, T] \rightarrow \mathbb{R}$ is given by

$$
L_{\alpha}(z, t)=\phi(z, t)+u^{*}(t)^{\prime} h(z, t)+\frac{\alpha}{2}\|h(z, t)\|^{2} .
$$

By differentiation of $L_{\alpha}$ we obtain

$$
\nabla L_{\alpha}(z, t)=\nabla \phi(z, t)+\nabla h(z, t)\left[u^{*}(t)+\alpha h(z, t)\right]
$$

and

$$
\begin{aligned}
\nabla^{2} L_{\alpha}(z, t) & =\nabla^{2} \phi(z, t)+\sum_{i=1}^{p}\left[u_{i}^{*}(t)+\alpha h_{i}(z, t)\right] \nabla^{2} h_{i}(z, t) \\
& +\alpha \nabla h(z, t)^{\prime} \nabla h(z, t) .
\end{aligned}
$$

Let's check that the hypotheses of Proposition 2.1 hold for the problem (6) at $\bar{z}$. Indeed, by $(\mathrm{H} 1)$ and $(\mathrm{H} 2)$ we have that $L_{\alpha}(\cdot, t)$ is continuously differentiable throughout $[0, T]$, $L_{\alpha}(z, \cdot)$ and $\nabla L_{\alpha}(z, \cdot)$ are Lebesgue measurable for each $z$ and as

$$
\left\|\nabla L_{\alpha}(\bar{z}(t), t)\right\|=\left\|\nabla \phi(\bar{z}(t), t)+\nabla h(\bar{z}(t), t) u^{*}(t)\right\|=0 \text { a.e. } t \in[0, T],
$$


it result that (H1) holds for the problem (6) and as $\nabla L_{\alpha}(\bar{z}(t), t)=0$ a.e. $t \in[0, T]$, the assumption (a) of Proposition 2.1 holds. Now, if $\gamma \in N \backslash\{0\}$,

$$
\begin{aligned}
& \int_{0}^{T} \gamma(t)^{\prime}\left\{\nabla^{2} L_{\alpha}(\bar{z}(t), t)\right\} \gamma(t) d t \\
= & \int_{0}^{T} \gamma(t)^{\prime}\left\{\nabla^{2} \phi(\bar{z}(t), t)+\sum_{i=1}^{p} u_{i}^{*}(t) \nabla^{2} h_{i}(\bar{z}(t), t)+\alpha \nabla h(\bar{z}(t), t)^{\prime} \nabla h(\bar{z}(t), t)\right\} \gamma(t) d t \\
= & \int_{0}^{T} \gamma(t)^{\prime} \nabla^{2} L(\bar{z}(t), t) \gamma(t) d t+\alpha \int_{0}^{T} \gamma(t)^{\prime} \nabla h(\bar{z}(t), t)^{\prime} \nabla h(\bar{z}(t), t) \gamma(t) d t .
\end{aligned}
$$

From assumption $(i i)$ we have that

$$
\int_{0}^{T} \gamma(t)^{\prime} \nabla^{2} L(\bar{z}(t), t) \gamma(t) d t<0
$$

for all $\gamma \in N \backslash\{0\}$. By applying Lemma 3.1 with $R(t)=\nabla^{2} L(\bar{z}(t), t)$ and $S(t)=$ $\nabla h(\bar{z}(t), t)^{\prime} \nabla h(\bar{z}(t), t)$, it follows that there exists $\bar{\alpha}>0$ such that

$$
\int_{0}^{T} \gamma(t)^{\prime}\left\{\nabla^{2} L_{\alpha}(\bar{z}(t), t)\right\} \gamma(t) d t<0
$$

for all $\gamma \in L_{\infty}\left([0, T] ; \mathbb{R}^{n}\right) \backslash\{0\}$ and $\alpha>\bar{\alpha}$. Choosing an $\alpha>\bar{\alpha}$, the assumptions of Proposition 2.1 are satisfied and hence $\bar{z}$ is a local optimal solution of problem (6), that is, there exists $\epsilon>0$ such that $\tilde{P}(\bar{z}) \geq \tilde{P}(z)$ for all $z$ satisfying $\|z-\bar{z}\|_{\infty}<\epsilon$. Since for all $z \in \Omega$, we have that $h(z(t), t)=0$ a.e. $t \in[0, T]$, then $L_{\alpha}(z(t), t)=L(z(t), t)$ and $\bar{z} \in \Omega$ implies in $h(\bar{z}(t), t)=0$ a.e. $t \in[0, T]$, we obtain

$$
P(\bar{z})=\tilde{P}(\bar{z}) \geq \tilde{P}(z)=P(z)
$$

for all $z \in \Omega$ with $\|z-\bar{z}\|_{\infty}<\epsilon$. Thereby, $\bar{z}$ is a local optimal solution for problem (1).

Below, we present an illustrative example where we use Theorem 3.1 to obtain sufficient optimality conditions for a certain problem.

Example 3.1. Consider the following problem in $L_{\infty}\left([0,1] ; \mathbb{R}^{3}\right)$ :

$$
\begin{array}{ll}
\text { maximize } & P(z)=\int_{0}^{1}\left[z_{1}(t) z_{2}(t)+z_{2}(t) z_{3}(t)+z_{1}(t) z_{3}(t)\right] d t \\
\text { subject to } & z_{1}(t)+z_{2}(t)+z_{3}(t)=3 \text { a.e. } t \in[0,1] .
\end{array}
$$

Note that $\bar{z}(t)=(1,1,1)^{\prime} \quad$ a.e. $t \in[0,1]$ and $\bar{u}(t)=-2$ a.e. $t \in[0,1]$ satisfy

$$
\left(\begin{array}{l}
z_{2}(t)+z_{3}(t) \\
z_{1}(t)+z_{3}(t) \\
z_{1}(t)+z_{2}(t)
\end{array}\right)+u(t)\left(\begin{array}{l}
1 \\
1 \\
1
\end{array}\right)=0 \quad \text { a.e. } t \in[0,1] .
$$

For $\gamma \in N \backslash\{0\}$, the condition (ii) in Theorem 3.1 also happens:

$$
\int_{0}^{1} \gamma(t)^{\prime}\left(\begin{array}{lll}
0 & 1 & 1 \\
1 & 0 & 1 \\
1 & 1 & 0
\end{array}\right) \gamma(t) d t=\int_{0}^{1}-\gamma_{1}^{2}(t)-\gamma_{2}^{2}(t)-\gamma_{3}^{2}(t) d t<0
$$

Therefore, from Theorem 3.1 we see that $\bar{z}$ is a local optimal solution for problem (7). 


\section{Final Comments}

In this work, we present sufficient optimality conditions for continuous-time optimization problem with equality constraints. Obtaining sufficient optimality conditions when inequality constraints are present, without using hypotheses of generalized convexity, is going to be a topic of future work.

\section{References}

[1] R. Bellman. Bottleneck problems and dynamic programming, Proceedings of the $\mathrm{Na}$ tional Academy of Sciences of the United States of America, 39:947-951, 1953.

[2] A. J. V. Brandão, M. A. Rojas-Medar and G. N. Silva. Nonsmooth continuous-time optimization problems: sufficient conditions, Journal of Mathematical Analysis and Applications, 227(2):305-318, 1998. DOI: 10.1006/jmaa.1998.6024.

[3] A. J. V. Brandão, M. A. Rojas-Medar and G. N. Silva. Nonsmooth continuous-time optimization problems: necessary conditions. Computers $\& 3$ Mathematics with Applications, 41(12):1477-1486, 2001. DOI: 10.1016/S0898-1221(01)00112-2.

[4] L. C. Evans. Weak convergence methods for nonlinear partial differential equations. American Mathematical Society, n. 74, 1990.

[5] L. A. Lusternik and V. J. Sobolev. Elements of Functional Analysis. Frederick Ungar, New York, 1961.

[6] V. A. de Oliveira and M. A. Rojas-Medar. Continuous-time optimization problems involving invex functions, Journal of Mathematical Analysis and Applications, 237(2):1320-1334, 2007.

[7] T. W. Reiland, Optimality Conditions and Duality in Continuous Programming I. Convex Programs and a Theorem of the Alternative. Journal of Mathematical Analysis an Applications, 77:297-325, 1980.

[8] G. J. Zalmai, The Fritz John and Kuhn-Tucker optimality conditions in continuous time nonlinear programming, Journal of Mathematical Analysis and Applications, 110:503-518, 1985.

[9] G. J. Zalmai, Sufficient optimality conditions in continuous-time nonlinear programming, Journal of Mathematical Analysis and Applications, 111:130-147, 1985. 